\title{
REPRESENTACIONES MENTALES, LENGUAJES Y CÓDIGOS EN LA ENSEÑANZA DE CIENCIAS NATURALES. UN EJEMPLO PARA EL APRENDIZAJE DEL CONCEPTO DE REACCIÓN QUÍMICA A PARTIR DEL CONCEPTO DE MEZCLA
}

\author{
GALAGOVSKY, LYDIA R. ${ }^{1,2}$, RODRÍGUEZ, MARÍA ALEJANDRA ${ }^{2}$, STAMATI, NORA ${ }^{2}$ \\ y MORALES, LAURA F. ${ }^{2}$ \\ ${ }^{1}$ Centro de Formación e Investigación en Enseñanza de las Ciencias \\ Facultad de Ciencias Exactas y Naturales. Universidad de Buenos Aires \\ Ciudad Universitaria, Pabellón II. 1428 Buenos Aires. Argentina \\ ${ }^{2}$ Universidad Nacional de General San Martín, Peatonal 52. 3568 (1560) Provincia de Buenos Aires. Argentina \\ lyga@qo.fcen.uba.ar
}

\begin{abstract}
Resumen. Las propuestas formuladas por Johnstone acerca del uso de tres niveles de representaciones mentales para quien enseña química y de un modelo sencillo de aprendizaje basado en la importancia de los aprendizajes previos son el marco teórico del presente trabajo. Nuestro objetivo se centró en indagar la adquisición del aprendizaje del concepto de reacción química a partir del concepto de mezcla, sobre dos grupos de alumnos diferentes, de 16-17 años. El trabajo nos permitió revisar críticamente el marco teórico, tomar conciencia de la diversidad de lenguajes que utilizamos durante la enseñanza y de la importancia de explicitar los códigos de cada lenguaje para mejorar la comunicación entre docentes y alumnos.

Palabras clave. Memoria, lenguajes, representaciones mentales, reacción química, mezcla.
\end{abstract}

Summary. Johnstone's suggestions concerning three levels for mental representations of the chemical thinking and a simple learning model based on the importance of the knowledge already achieved are the theoretical frame for the present work. Our aim was to investigate the acquisition of the concept of chemical reaction starting with the already known concept of mix, with two groups of students of 16-17 years old. The work done allowed us both to do a critical review of the theoretical frame and to be aware of the diversity of languages we use to teach. This last point showed us the importance of making explicit the codes within each language used in order to enhance communication between professors and students.

Keywords. Memory, languages, mental representation, chemical reaction, mix.

\section{INTRODUCCIÓN}

Las investigaciones y propuestas hechas por el profesor Johnstone $(1982,1991,1997,1999)$ concernientes a la enseñanza de las ciencias y, en particular, de la química, basadas en teorías de funcionamiento de la memoria, brindan una plataforma teórica muy interesante para elaborar y poner a prueba hipótesis relacionadas con procesos de enseñanza y aprendizaje de temas de química en el aula.
En el presente artículo mostramos una indagación de tipo cualitativo que nos permitió, por un lado, confirmar la afirmación de Johnstone que dice: «Lo que realmente sabemos y entendemos controla lo que aprendemos.»; y, por otro lado, reformular la propuesta de este autor acerca de los tres niveles de pensamiento que se requieren para saber química (Johnstone, 1991). 


\section{EL MARCO TEÓRICO}

\section{La memoria perceptiva y los aprendizajes previos con- dicionan lo que aprendemos}

La mayoría de los conceptos que nos son familiares tienen algo de tangible, algo que puede ser reconocido por nuestros sentidos. El aprendizaje de este tipo de conceptos nos resulta más sencillo que el de conceptos abstractos.

Johnstone (1991) plantea que la mayoría de los conceptos que se utilizan en química no tienen un medio sencillo y directo de ser percibidos por vía sensible. Cuando hablamos de elemento o compuesto, no tenemos una forma inmediata de hacer percibir estas ideas a los estudiantes. Ejemplos de elementos pueden ser polvos amarillos, gases incoloros o líquidos marrones, pero éstos también pueden ser ejemplos de compuestos o de mezclas. ¿Cuáles son sus puntos en común, cuáles son sus rasgos diferenciales? Para un experto, estos aspectos son evidentes, pero ¿son tan sencillos de comprender para un alumno que comienza a aprender química?

Conceptos tales como electrón, unión química, fotones, moléculas, etc. son ideas que están más allá de nuestros sentidos, y los alumnos no tienen experiencia previa que les facilite dar un significado preciso a estas palabras.

Las investigaciones sobre educación en química revelan que, aunque demuestran cierta habilidad en aprobar los exámenes, los estudiantes evidencian errores conceptuales difíciles de superar (Ben Zvi, 1992; Taber, 1998).

Para poder hacer inferencias acerca de posibles explicaciones sobre esta distancia entre lo que se enseña y lo que realmente aprenden los alumnos, Johnstone (1997, 1999) propone utilizar el modelo de aprendizaje que se muestra en la figura 1 partiendo de las siguientes premisas: a) Las percepciones que registramos a través de los sentidos no son objetivas, están filtradas e interpretadas en forma idiosincrática.

b) Lo que ya sabemos, lo que está guardado en nuestro banco de memoria a largo plazo (MLP) (Mayer, 1985), es lo que controla la significación que damos a las nuevas informaciones sensoriales que recibimos, es decir, condiciona nuestro filtro perceptivo.

c) Nuestra memoria de trabajo (MT) es esa parte de nuestra actividad mental que, conscientemente, presta atención a una situación dada y piensa sobre ella. Es la que se fija en la percepción que ha entrado y trata de otorgarle un sentido.

d) Dar sentido a algo es colocarlo, ubicarlo, agregarlo a algo ya conocido o a algo en que se cree.

e) La MT tiene dos funciones: una es sostener momentáneamente la información en el foco de la atención -en la memoria de corto plazo-y la otra es darle un formato a esa información para guardarla, utilizarla o decidir descartarla. Esta memoria de trabajo tiene una capacidad limitada, saturándose si la cantidad de información recibida es demasiada o si su procesamiento es demasiado complicado.

f) Una información que satura o sobrepasa la capacidad de MT de un sujeto no podrá ser procesada por él.

Cuando un alumno se encuentra por primera vez con conceptos de química, sus sentidos ya no le permiten aprender mediante la percepción directa. Los conceptos de la química son entes abstractos, mediados por interpretaciones simbólicas. Los docentes intentamos hacer dibujos, analogías, experiencias de laboratorio, además de dar definiciones, reglas, leyes y teorías, pero, aun así, estas acciones acaban provocando bases conceptuales erróneas o forzan-

Figura 1

Modelo de aprendizaje propuesto por Johnstone (1997) en que se muestra cómo los aprendizajes anteriores condicionan los nuevos.

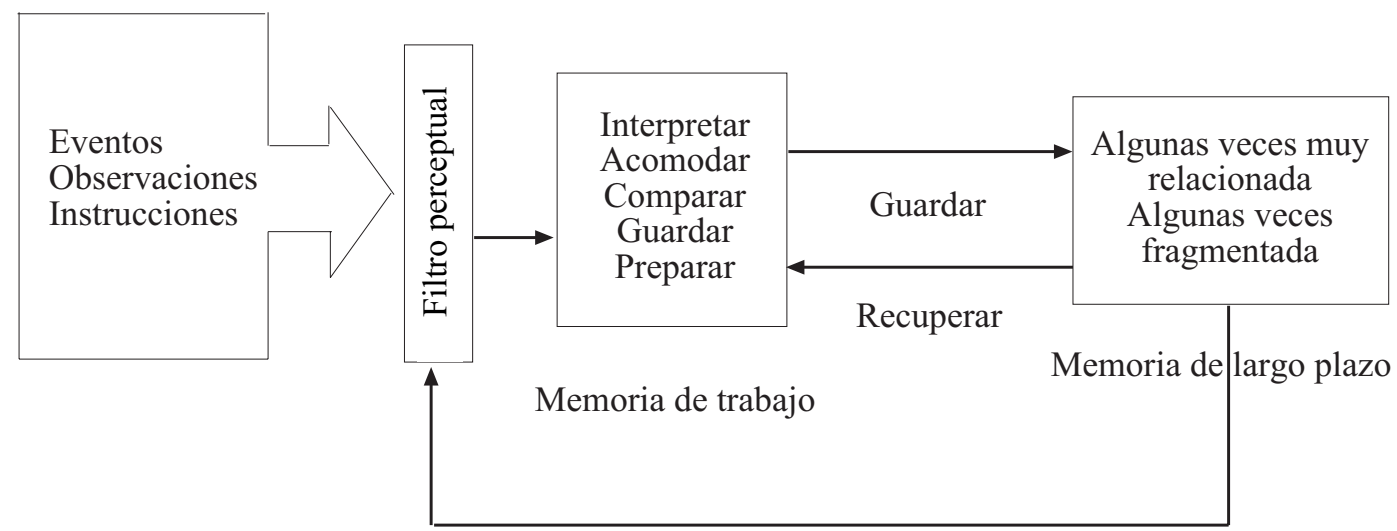

Circuito de retroalimentación del filtro perceptivo 
do a aprendizajes memorísticos, fragmentados e, incluso, sin sentido.

\section{Niveles de representación mental para los fenómenos naturales}

Johnstone $(1982,1991)$ propuso para las ciencias naturales, y para la química en particular, los niveles macroscópico, submicroscópico y simbólico de pensamiento, relacionados en el triángulo que se muestra en la figura 2.

El nivel macroscópico corresponde a las representaciones mentales adquiridas a partir de la experiencia sensorial directa. Este nivel se construye mediante la información proveniente de nuestros sentidos, basada en propiedades organolépticas, visuales, auditivas y táctiles. Todos los sistemas materiales que manipulamos podemos caracterizarlos mediante descripciones sensoriales que aportan información a este nivel. Un vaso con un líquido, un vaso vacío o un vaso con un polvo se perciben de esa forma. Suponer o interpretar que el líquido puede estar puro o ser una solución, que el vaso vacío está lleno con una solución gaseosa (aire) o que el polvo es una mezcla imperceptible con una composición dada, no son percepciones directamente inferidas del nivel macroscópico.

El nivel submicroscópico, según Johnstone, hace referencia a las representaciones abstractas, modelos que tiene en su mente un experto en química asociados a esquemas de partículas. Ejemplos de este nivel son las imágenes de esferitas que solemos utilizar para describir el estado sólido de una sustancia pura, o sus cambios de estado, o sus transformaciones químicas, que se corresponden con una representación mental de lo que sucede según el modelo particulado de la materia.

El tercer nivel, el simbólico, involucraría formas de expresar conceptos químicos mediante fórmulas, ecuaciones químicas, expresiones matemáticas, gráficos, definiciones, etc.

Según este triángulo, un experto en química que piensa en la reacción entre el nitrato de plata y el cloruro de sodio,

Figura 2

Niveles representacionales en química, según Johnstone (1982).

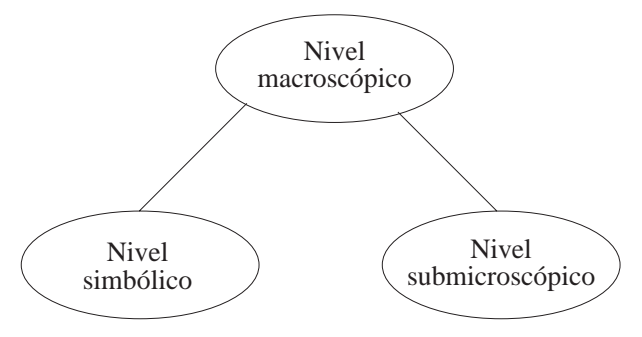

ambos en solución acuosa, estaría manejando simultáneamente los tres niveles de pensamiento. Por ejemplo:

En el nivel macroscópico, el experto sabe que al combinar ambos líquidos incoloros y transparentes se produce un precipitado blanco. Simultáneamente, puede pensar en el nivel submicroscópico y describir la reacción mediante un esquema de partículas como esferitas, en el que se representan reactivos, productos y, eventualmente, el solvente. Estos esquemas de partículas utilizan formas y colores que otro experto - otro profesor-podría identificar fácilmente porque puede reconstruir mentalmente el sistema apropiado. El alumno, en cambio, puede creer que así se «ven» los átomos, incluso con esos colores.

Finalmente, en el nivel simbólico podríamos relatar lo ocurrido con palabras, o mediante fórmulas, por ejemplo, escribiendo:

$$
\begin{gathered}
\mathrm{AgNO}_{3}+\mathrm{NaCl} \rightarrow \mathrm{AgCl}+\mathrm{NaNO}_{3} \\
\text { o bien } \\
\mathrm{Ag}^{1+}(\mathrm{aq})+\mathrm{NO}_{3}{ }^{1-}(\mathrm{aq})+\mathrm{Na}^{1+}(\mathrm{aq})+\mathrm{Cl}^{1-}(\mathrm{aq}) \\
\rightarrow \mathrm{AgCl}(\mathrm{s})+\mathrm{NaNO}_{3}(\mathrm{aq})
\end{gathered}
$$

Un docente que está explicando este fenómeno químico a sus alumnos está pensando en los tres niveles representacionales propuestos por Johnstone, simultáneamente, aunque en su discurso sólo explicita información en cada uno de ellos alternativa y secuencialmente. El docente, en general, no es consciente de la demanda real que debe soportar la memoria de trabajo de los alumnos, para procesar la información que está recibiendo, ni de que para ellos los dibujos y explicaciones no tienen anclaje directo en la percepción macroscópica del fenómeno.

Las investigaciones de otros autores reforzarían los problemas planteados por Johnstone, en el sentido de que los alumnos no manejan simultáneamente los niveles representacionales indicados, al intentar explicar un fenómeno químico. Boujaude (1991) estudió la comprensión de la combustión y Gabriela y otros (1990) estudiaron la comprensión de reacciones químicas y procesos espontáneos y encontraron que los estudiantes tienden a explicar fenómenos químicos usando, fundamentalmente, un criterio visual relacionado con las propiedades macroscópicas.

Asimismo, la comprensión de los estudiantes acerca de las fórmulas químicas y de las ecuaciones está muy relacionada con la conceptualización que hayan hecho sobre las partículas. Numerosos estudios (Ben Zvi, Eylon y Siberstein, 1982; Yarroch, 1985; Maloney y Friedel, 1991; Friedel y Maloney, 1992) indican que los estudiantes de química de nivel secundario no asocian las fórmulas químicas con una apropiada representación de nivel particulado, muestran dificultad en relacionar el subíndice de las fórmulas químicas con el número apropiado de átomos en dibujos que representaban las partículas o cuando se les pedía que las dibujaran ellos. Yarroch demostró que los estudiantes no distinguen entre los coeficientes que preceden a las fórmulas y los subíndices que indican atomicidad. Niaz y Lawson (1985) también encontraron que, incluso entre los estudiantes que eligieron química 
como asignatura complementaria, se ponía de manifiesto la dificultad para ajustar ecuaciones.

Todos estos autores coinciden en que las ideas sobre la discontinuidad de la materia, sobre que sus partículas están en movimiento y que una colección de esas partículas tiene propiedades diferentes de las de una sola de dichas partículas, no son ideas intuitivas. Estas y otras ideas relativas a las propiedades de la materia son muy abstractas y, consecuentemente, difíciles de entender para los alumnos.

\section{OBJETIVOS DE LA INDAGACIÓN}

El objetivo de nuestra investigación fue el de poner a prueba la aplicabilidad del triángulo de Johnstone durante el proceso de aprendizaje de química, centrándonos en la construcción del concepto de reacción química.

Las preguntas que guiaron la investigación fueron:

1) ¿Cuáles son los niveles representacionales de la química más accesibles para los alumnos?

2) ¿Cuáles son las dificultades u obstáculos epistemológicos que entorpecen la movilidad entre los niveles representacionales?

3) ¿Son los niveles representacionales de Johnstone (1982) aplicables a una investigación sobre aprendizaje, o son sólo aplicables como sugerencias para la enseñanza?

4) ¿Qué tipo de acciones didácticas favorecerían la movilidad entre niveles representacionales?

Dado que nos centrarnos en el aprendizaje del concepto reacción química en alumnos de 16-17 años que ven química por primera vez en la escuela, y dada la complejidad de este concepto (Solsona et al., 1998, 2001), los objetivos específicos de este trabajo fueron:

1) Detectar las representaciones mentales de los alumnos acerca de la experiencia de producir una reacción química exclusivamente a partir del calentamiento de una mezcla (caso azufre y limaduras de hierro).

2) Analizar la validez de la aplicación de los tres niveles de representación de Johnstone en alumnos que deben aprender el concepto de reacción química por primera vez.

\section{DESCRIPCIÓN DE LA EXPERIENCIA}

\section{Metodología}

La indagación fue de tipo cualitativo, según un paradigma interpretativo con diseño descriptivo. Este diseño nos permitió recoger información, identificar dificultades, realizar comparaciones y evaluaciones y proponer posibles cambios en la práctica pedagógica del campo de la química en particular. En este sentido, la muestra de alumnos seleccionada no se considerará representativa del universo de alumnos que cursan química en el nivel seleccionado. De ningún modo pretendemos llegar a abstracciones generales de carácter universal, ya que concebimos la realidad educativa como múltiple e intangible; por lo tanto, la investigación en este campo tenderá a diverger y no podrá determinarse una única verdad, ni será posible la predicción ni el control (Colás Bravo y Buendía Eisman, 1994).

La propia docente de las clases recogió los datos, basados en la observación con registro simultáneo y posterior de los hechos ocurridos en clase, y las respuestas a los cuestionarios. Todas las actividades realizadas por los alumnos admitieron la discusión grupal, pero las producciones escritas fueron siempre individuales. El análisis de las respuestas de los alumnos permitió categorizarlas e indicar sus porcentajes, aunque ello no revistió en sí mismo un interés cuantitativo.

\section{Muestra}

El estudio se realizó con dos grupos de alumnos (Rodríguez, 2001): el primero, denominado grupo $A$, formado por 20 alumnas que cursaban $4^{\circ}$ año de bachillerato; el segundo grupo, denominado grupo $B$, estaba conformado por 48 alumnos varones que cursaban el tercer año de escuela técnica con orientación electromecánica ${ }^{1}$. Para ambos grupos éste era su primer curso de química y asistían a tres clases semanales de química de 40 minutos cada una. En el momento de iniciar la enseñanza del tema de reacciones químicas, habían desarrollado idéntica secuencia de contenidos: materia, estados y cambios de estado; modelo cinéticomolecular; fenómenos físicos y químicos; mezcla heterogénea y homogénea; métodos de separación y fraccionamiento; sustancias simples y compuestas; estructura atómica: configuración electrónica; iones; tabla periódica; propiedades periódicas: electronegatividad y electropositividad; enlaces químicos: iónico, covalente, metálico; estructuras de Lewis; nomenclatura y fórmulas de óxidos.

\section{Actividades}

\section{- Actividad 1}

Cada grupo de cuatro alumnos recibió dos vidrios de reloj, uno con azufre en polvo y otro con limaduras de hierro, una varilla de vidrio, un imán, un mortero con pilón y un cuestionario (Cuadro I) cuya respuesta debía ser individual.

\section{- Actividad 2}

Una vez respondidas las preguntas 1 a 5 , los alumnos recibían un material adicional: un mechero, un trípode, una tela metálica, una gradilla, un tubo de ensayo, una pinza de madera, un vaso de precipitados con agua, una espátula. Luego, se les solicitó que contestaran las preguntas 6 a 8 (Cuadro I), siempre con discusión grupal pero con respuestas individuales. 
Cuadro I

\section{Cuestionario de la actividad 1}

1) Si se colocan el azufre y el hierro en el mismo vidrio de reloj. ¿Qué se obtiene?

2) ¿Cómo de podría esquematizar lo obtenido?

3) ¿Podrían separarse nuevamente el azufre del hierro? ¿Cómo?

4) En caso de responder afirmativamente a la pregunta anterior, ¿por qué?

5) ¿Es posible producir una reacción química entre el azufre y el hierro? ¿Por qué?

\section{Cuestionario de la actividad 2}

Teniendo en cuenta el siguiente material: un mechero, un trípode, una tela metálica, una gradilla, un tubo de ensayo, una pinza de madera, un vaso de precipitados y una espátula; y empleándolo según consideres más apropiado, responde por escrito:

6) ¿Cómo puede procederse para verificar lo respondido en la pregunta anterior? (Pregunta 5)

7) Caracterice y fundamente el fenómeno producido.

8) Realice un esquema que muestre el fenómeno producido al calentar una mezcla de azufre y hierro.

\section{Objetivos de las actividades}

Nuestra expectativa era que la mayoría de los alumnos recordaría que la mezcla de polvo de azufre y limaduras de hierro tiene la posibilidad de separarse mediante el uso de un imán, ya que esto había sido enseñado unas diez clases antes, al tratar el tema de sistemas heterogéneos de mezclas sólidas.

Responder a los cuestionarios tenía tres objetivos principales:

1) Para las preguntas 1 a 4: indagar los conocimientos previos y las representaciones mentales de los alumnos respecto a qué es una mezcla heterogénea y cómo representarla a nivel submicroscópico.

2) Para la pregunta 5: indagar sobre obstáculos epistemológicos en la adquisición del concepto de cambio químico.

3) Para las preguntas 6-8: indagar la facilidad o dificultad en reconocer conceptualmente la diferencia entre una mezcla y una reacción química, y los niveles representacionales de Johnstone -macroscópico, submicroscópico y simbólico- disponibles para diferenciar, expresar y simbolizar dichos cambios.

\section{ANÁLISIS DE LOS RESULTADOS PARA LA ACTIVIDAD 1}

\section{Respuestas a las preguntas 1-4 para el grupo A}

A continuación desglosaremos el análisis de las respuestas de las alumnas a cada pregunta del cuestionario de la actividad 1 .

Pregunta 1: Si colocan el azufre y el hierro en el mismo vidrio de reloj, ¿qué se obtiene?

Todas las alumnas respondieron que el sistema era heterogéneo porque presentaba fases. La respuesta evidenciaba que las alumnas poseían conocimiento sobre sistemas heterogéneos. Este tema había sido trabajado en las clases anteriores desde varios ejemplos, incluido el de la mezcla de hierro y azufre.

Pregunta 2: ¿Cómo podrían esquematizar lo obtenido?

El 33\% de las alumnas realizó un esquema donde se mostraba un color amarillo difuso, esparcido y puntitos o rayitas negras dispersadas en la masa de color amarillo. Este dibujo mostró claramente que las alumnas representaron lo percibido por sus sentidos: las características del nivel macroscópico de la mezcla en cuestión.

Un 55\% de las alumnas empleó en sus esquemas un símbolo gráfico para el azufre y otro para el hierro: triángulos y rayitas, o círculos y cruces, etc.; no agregaron color ni explicación alguna para los símbolos utilizados. Entendemos que este tipo de dibujos se refieren a la representación de un nivel submicroscópico -según Johnstone- del sistema, eligiendo un código de dos formas diferentes para mostrar las partículas que no se ven a simple vista.

Un $12 \%$ de las alumnas realizó esquemas donde se superponían rasgos de los dibujos anteriores: utilizando dos formas diferentes para simbolizar las partículas de azufre y de hierro pero, además, al azufre lo pintaron de amarillo y las partículas de hierro fueron bien remarcadas en color oscuro. Este tipo de representación no se corresponde exactamente con ninguno de los niveles propuestos por Johnstone. Son los trabajos de Driver $(1981,1992)$ los que dan mejor cuenta de esta tendencia según la cual muchos alumnos aceptan el modelo particulado de la materia, pero en sus representaciones mentales adjudican a las partículas propiedades observables, características del nivel macroscópico ${ }^{2}$.

La existencia de este nuevo nivel representacional indicaría que, si bien el triángulo de Johnstone debería tenerse en cuenta al enseñar conceptos químicos -ya que el experto tiene movilidad mental entre los niveles macroscópicos, submicroscópico y simbólico-, debe reconocerse que los novatos, durante el proceso de aprendizaje de dichos conceptos, atravesarían por un nivel representacional intermedio, erróneo, entre el macroscópico y el submicroscópico. Este nuevo nivel, al que hemos denominado nivel de representación semiparticulado (Fig. 3), daría cuenta de una correspondencia entre la forma de representar aspectos perceptivos de un fenómeno y los conocidos trabajos so- 
Figura 3

Nivel de interpretación semiparticulado detectado durante el aprendizaje erróneo de conceptos químicos.

\begin{tabular}{|c|c|c|}
\hline $\begin{array}{c}\text { NIVEL } \\
\text { MACROSCÓPICO }\end{array}$ & $\begin{array}{l}\text { NIVEL SEMI- } \\
\text { PARTICULADO }\end{array}$ & $\begin{array}{c}\text { NIVEL SÚB- } \\
\text { MISCROSCÓPICO }\end{array}$ \\
\hline $\begin{array}{c}\text { Requiere, } \\
\text { simplemente, } \\
\text { el registro de datos } \\
\text { perceptibles por los } \\
\text { sentidos. } \\
\text { Se expresa mediante } \\
\text { lenguaje visual } \\
\text { y verbal }\end{array}$ & $\begin{array}{c}\text { Durante el } \\
\text { aprendizaje de } \\
\text { química existe } \\
\text { una tendencia } \\
\text { a otorgar rasgos } \\
\text { perceptibles } \\
\text { a entidades } \\
\text { - conceptos- } \\
\text { no perceptibles }\end{array}$ & $\begin{array}{c}\text { Requiere } \\
\text { conocimientos sobre } \\
\text { entidades no } \\
\text { perceptuales para } \\
\text { hacer una } \\
\text { interpretación } \\
\text { particular de la } \\
\text { materia. Se expresa } \\
\text { mediante un lenguaje } \\
\text { gráfico que utiliza } \\
\text { códigos específicos }\end{array}$ \\
\hline
\end{tabular}

bre ideas alternativas adquiridas durante la instrucción de los alumnos (Driver, 1992). Esta categorización aquí presentada se verá confirmada en el análisis de las respuestas a la pregunta 8 .

Pregunta 3: ¿Podrían separarse nuevamente el azufre del hierro? ¿Cómo?

Todas las alumnas respondieron «por medio del imán», poniendo de manifiesto lo que habían aprendido cuando estudiaron sistemas heterogéneos.

Pregunta 4: En caso de responder afirmativamente a la pregunta anterior, ¿por qué?

Las respuestas obtenidas y sus porcentajes se muestran en la tabla I. Se observa que todas las respuestas fueron correctas y, además, un $22 \%$ distinguió entre el concepto de mezcla y el concepto de cambio químico, que habían sido enseñados como introducción a la asignatura, en referencia al cambio físico ${ }^{3}$.

Tabla I

Respuestas de las alumnas del grupo A a la pregunta 4.

\begin{tabular}{|l|c|}
\hline Respuesta & Porcentaje \\
\hline El hierro tiene propiedades ferromagnéticas. & $22 \%$ \\
$\begin{array}{l}\text { El hierro tiene propiedades ferromagnéticas } \\
\text { y el azufre no. }\end{array}$ & $56 \%$ \\
$\begin{array}{l}\text { El hierro es atraído por el imán porque } \\
\text { tiene propiedades ferromagnéticas, } \\
\text { el azufre no, y no hubo cambio químico. }\end{array}$ & $22 \%$ \\
\hline
\end{tabular}

Con las respuestas obtenidas en las primeras cuatro preguntas inferimos que las alumnas conocían el concepto de mezcla. Sin embargo, mientras en las respuestas 1,3 y 4 que requerían un lenguaje de tipo verbal, el éxito fue de un $100 \%$, en la respuesta 2 , que demandó la realización de un esquema, sólo un 55\% de las alumnas se expresó mediante el nivel representacional submicroscópico esperado.

Este resultado nos sugiere fuertemente que el término esquematizar no tiene el mismo significado para expertos y novatos. Para la docente, esquematizar era sinónimo de «dibujar el sistema estudiado utilizando exclusivamente códigos donde se distinguieran las partículas, que no pueden ser observadas a simple vista»; pero para el $45 \%$ de las alumnas, esquematizar remitió a dibujar aspectos macroscópicos de la experiencia observada.

Una conclusión importante, que se desprende de estos resultados, es el hecho de que las interpretaciones a nivel submicroscópico de fenómenos macroscópicos no son intuitivas para los alumnos.

Desde nuestra perspectiva, proponemos que el nivel submicroscópico es, en sí mismo, un nivel simbólico. En la figura 4 mostramos esta perspectiva que modifica la recomendación de Johnstone respecto de la existencia de tres niveles representacionales para la química (Fig. 2) proponiendo la existencia de sólo dos niveles representacionales -el macroscópico y el simbólico- y la consideración de que el nivel submicroscópico de Johnstone es, en realidad, un caso especial de nivel simbólico que interpreta explicaciones mediante esquemas de partículas, expresándose a través de un lenguaje gráfico que utiliza códigos específicos. Esta perspectiva puede aclararse con un ejemplo como el que sigue.

Para explicar cambios de estado de la materia, los docentes recurrimos a dibujos de esferitas para representar las 
partículas involucradas en los procesos de fusión y ebullición. La separación entre esferitas nos permite mostrar cómo varían las distancias intermoleculares en cada uno de los estados. Cada esferita equivaldría, por ejemplo, a una molécula de agua.

En otro momento, para explicar la formación de moléculas, podemos dibujar esferitas como equivalentes a diferentes átomos y no ya a una molécula como en el caso anterior.

Finalmente, en un tercer momento, haciendo esquemas sobre solubilidad de una red cristalina iónica, podremos dibujar esferitas para mostrar la disposición de cationes y aniones en dicha red; en este caso, las esferitas equivaldrían a iones.

En este ejemplo mostramos que, generalmente, los docentes usamos esferitas para esquematizar especies químicas diversas, con un código diferente cada vez. Los docentes, como expertos, entendemos perfectamente la equivalencia de las esferitas en cada caso; sin embargo, es probable que los alumnos no comprendan fácilmente estos cambios de códigos.

\section{Respuestas a las preguntas 1-4 del grupo B}

El análisis de las respuestas es el siguiente:

\section{Figura 4}

Reconsideración del triángulo de Johnstone (Fig. 2): el nivel submicroscópico se considera ahora como un caso especial de representaciones mediante esquemas de partículas y se incluye en el nivel simbólico.

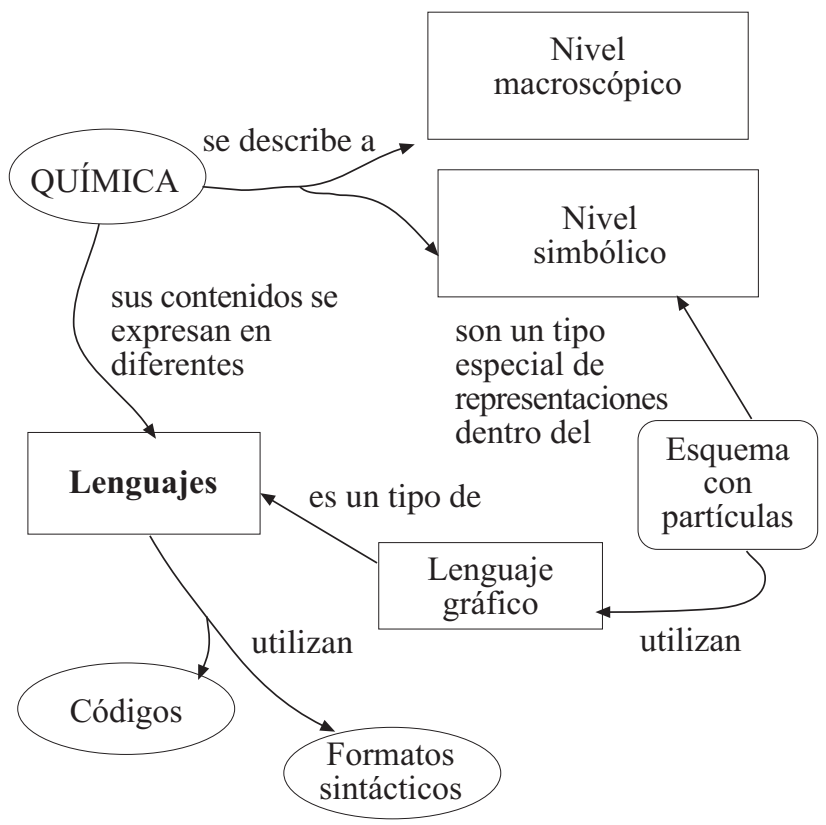

Tabla II

Respuestas de las alumnas del grupo A a la pregunta 5 .

\begin{tabular}{|l|c|}
\hline Respuestas & Porcentaje \\
\hline $\begin{array}{l}\text { No es posible porque, al acercar el imán, } \\
\text { el hierro vuelve a su estado original. } \\
\text { Cada sustancia conserva sus propiedades } \\
\text { y se diferencian sus partículas a simple vista. }\end{array}$ & $44 \%$ \\
\hline No se puede producir un cambio de sustancia. & $22 \%$ \\
\hline No, porque lo que hicimos fue un cambio físico. & $17 \%$ \\
\hline $\begin{array}{l}\text { Sí, porque el azufre es un no-metal y el hierro } \\
\text { es un metal. Pueden formar un enlace iónico. } \\
\text { En este caso no sería porque no hubo cambio } \\
\text { químico. }\end{array}$ & $17 \%$ \\
\hline
\end{tabular}

Figura 5

El $91 \%$ de los alumnos del grupo B respondió a la pregunta 2 desde el nivel submicroscópico (Johnstone), utilizando tres tipos de códigos.

Figura $5 a$

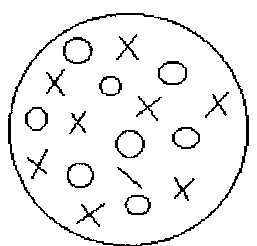

(47\%)

Figura $5 b$

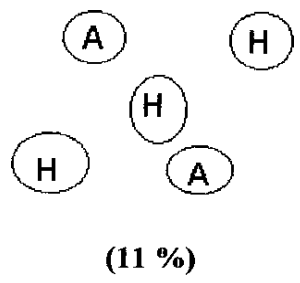

Figura 5c

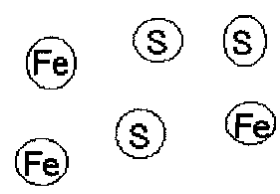

(Fe)

(33\%) 
Pregunta 1: Si colocan el azufre y el hierro en el mismo vidrio de reloj, ¿qué se obtiene?

Las respuestas fueron $100 \%$ correctas y, al igual que en el grupo A, apelaban al concepto de fase (un 78\%); un 22\% hizo referencia a que «cada sustancia conserva sus propiedades»».

Pregunta 2: ¿Cómo podría esquematizar lo obtenido?

A diferencia del grupo A, ningún alumno de este grupo utilizó el nivel macroscópico para esquematizar el sistema.

Un 9\% utilizó el nivel que hemos denominado semiparticulado (Fig. 3). El 91\% de los alumnos se inclinó por realizar esquemas de partículas (considerados antes como nivel submicroscópico, según Johnstone), utilizando códigos diferentes, según se muestra en la figura 5 . El 47\% de ellos empleó códigos geométricos dibujando círculos para el azufre y cruces para el hierro (Fig. 5a). Un 11\% dibujó una $\mathrm{A}$ encerrada en un círculo para indicar partículas de azufre, y una $\mathrm{H}$ encerrada en un círculo para indicar partículas de hierro. Es decir, eligieron un código que se corresponde con la primera letra del nombre de cada sustancia para identificar cada partícula de la misma (Fig. 5b). El $33 \%$ restante prefirió utilizar los símbolos de los elementos químicos, encerrándolos en un círculo (Fig. 5c).

Figura 6

Niveles de interpretación y lenguajes utilizados complementariamente por un experto en química.

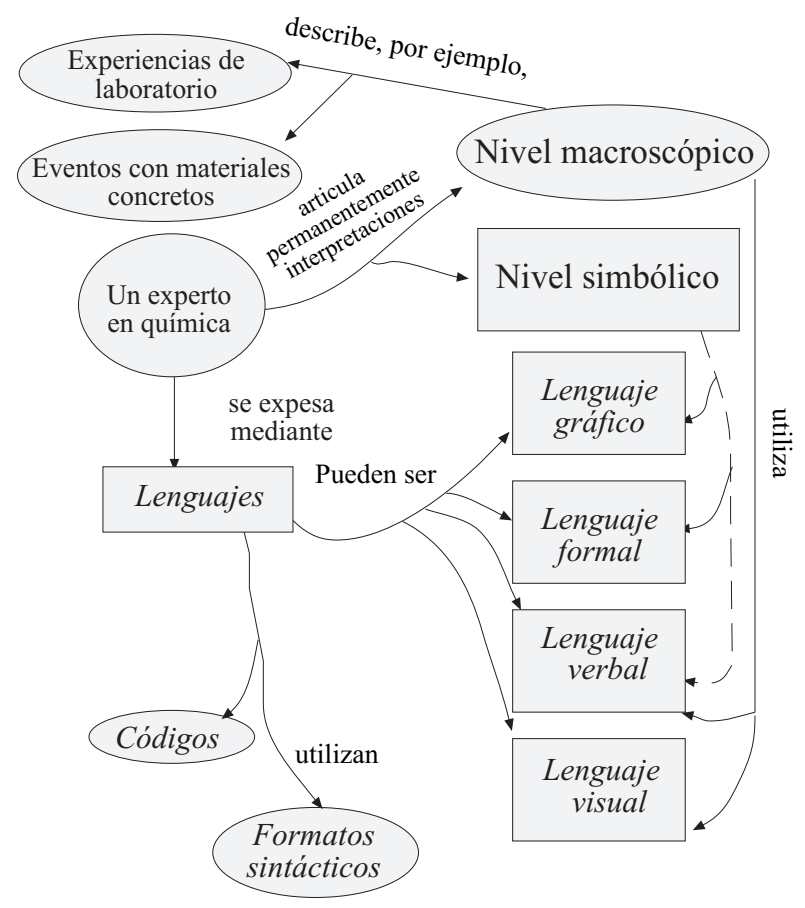

Las respuestas indicaron que, para este grupo de alumnos, existe mayor consenso en el significado de representación a nivel de esquemas de partículas para la consigna «esquematizar». El hecho de que estos alumnos mostraran una gran variedad de códigos para expresarse refuerza nuestra crítica al triángulo de la figura 2 y la necesidad de incluir este nivel submicroscópico como un lenguaje gráfico particular, dentro del nivel simbólico, según mostramos en la figura 4.

Pregunta 3: ¿Podría separar el azufre del hierro? ¿Cómo?

Los alumnos unánimemente contestaron: «por medio del imán». Entendemos que con esta respuesta estarían ratificando su conocimiento desde el nivel perceptual macroscópico.

Pregunta 4: En caso de responder afirmativamente a la pregunta anterior, ¿por qué?

Todos respondieron: «Porque el hierro tiene propiedades ferromagnéticas».

Se observaron coincidencias en las respuestas de tipo verbal entre los alumnos de ambos grupos, en tanto que la mayor diferencia se encontró al solicitar expresiones mediante lenguaje gráfico, tal como mostramos en la figura 5.

\section{SUGERENCIAS DIDÁCTICAS A PARTIR DE CONCLUSIONES PARCIALES}

Los análisis expresados hasta aquí permiten elaborar las siguientes propuestas didácticas.

- Los alumnos tienden a expresarse con más seguridad y con menos errores mediante el lenguaje verbal, probablemente debido a que este tipo de lenguaje es el más usado en clase, en sus variantes oral y escrita (en explicaciones, apuntes y textos). La tendencia a expresarse con un lenguaje verbal correcto puede ocultar aprendizajes exclusivamente memorísticos.

Debemos tener en cuenta que los docentes, como expertos en la disciplina, articulamos permanentemente descripciones a nivel macroscópico (tales como experiencias de laboratorio), expresadas mediante lenguaje verbal y visual, con explicaciones que las interpretan en un nivel simbólico. Tales explicaciones utilizan variados lenguajes además del verbal, por ejemplo, el lenguaje gráfico (para representar esquemas de partículas o bien variables en coordenadas de reacción o en ejes cartesianos, etc.). Otro tipo de lenguaje es aquél que utiliza fórmulas, ya sean éstas matemáticas o químicas; podríamos denominarlo lenguaje formal. Así, las explicaciones del experto utilizan complementariamente distintos lenguajes, como se muestra en la figura 6. Los lenguajes están caracterizados por utilizar códigos y formatos sintácticos convencionales y consensuados.

Es importante estimular a los alumnos para que se expresen también mediante otros tipos de lenguajes, además del 
verbal. Si el cruce de expresiones en lenguajes diferentes muestra incoherencias éstas pueden estar evidenciando errores en el aprendizaje.

- Los alumnos suelen otorgar propiedades macroscópicas a las partículas de nivel atómico o molecular (Fig. 3), tal como pintar de amarillo las partículas del elemento azufre. Esta tendencia se hace más evidente al solicitar representaciones de tipo esquemático que al solicitar explicaciones mediante lenguaje verbal. Los alumnos menos familiarizados con los diferentes niveles representacionales de la química parecen más expuestos a cometer este tipo de errores. Para superar esta tendencia es importante estimular a los alumnos a expresar sus representaciones mentales en diferentes tipos de lenguajes simbólicos, recreando consensos en cuanto a los códigos a utilizar.

- Los alumnos suelen memorizar aprendizajes en compartimentos estancos, caracterizados por lenguajes independientes y excluyentes. Para superar esta tendencia debemos estimular la toma de conciencia acerca de la naturaleza macroscópica de las experiencias químicas y sus múltiples interpretaciones y explicaciones a nivel simbólico, que pueden ser expresadas mediante una amplia variedad de lenguajes con códigos particulares (Fig. 6).

\section{ANÁLISIS DE RESULTADOS DE LA ACTIVI- DAD 2}

\section{Respuestas a las preguntas 5-8 del grupo A}

Como mencionamos más arriba, las respuestas a las preguntas 1 a 4 confirmaron que las alumnas habían aprendido el concepto de mezcla y reconocían perfectamente el caso «hierro y azufre» como una mezcla sólida separable. Estos prerrequisitos conceptuales podrían funcionar como conceptos inclusores (Galagovsky, 1999; Galagovsky y Muñoz, 2002) para procesar la nueva información referida a una reacción química entre el hierro y el azufre. Las preguntas $5 \mathrm{y}$ subsiguientes intentaron generar conflicto cognitivo (Galagovsky, 1993) sobre dicho concepto de mezcla, ya consolidado en la estructura cognitiva de las alumnas ${ }^{4}$.

Pregunta 5: Es posible producir una reacción química entre el azufre y el hierro? ¿Por qué?

E1 83\% de las respuestas obtenidas fue negativo. Las explicaciones dadas se resumen en la tabla II.

Sólo un $17 \%$ de las alumnas relacionó la pregunta con conocimientos teóricos anteriores sobre enlace químico y emitió hipótesis sobre la posibilidad de ocurrencia de un fenómeno químico, sin mencionar condición alguna para que ello ocurriera.

La posibilidad de imaginar una nueva variable, como la energía térmica, que diera origen a una transformación química pareció ser contraintuitiva.
Luego de recibir el material adicional (cuadro I), las respuestas a las preguntas 6 a 8 fueron:

Pregunta 6: ¿Cómo procederían para verificar lo respondido en la actividad anterior?

Dos subgrupos advirtieron la posibilidad de utilizar el mechero y probaron calentar el sistema de hierro y azufre, siendo uno de ellos el que había apelado a sus conocimientos sobre enlaces químicos en la respuesta a la pregunta 5 . Estos dos grupos verificaron que el hierro con propiedades magnéticas había desaparecido del sistema luego del calentamiento.

Dos grupos que habían asegurado la imposibilidad de producir una reacción química como respuesta a la pregunta anterior insistieron en triturar más finamente la mezcla de azufre y hierro en el mortero. Luego, una y otra vez, pasaban el imán verificando que el hierro era atraído. En virtud de esto contestaron en los respectivos informes: «Trituramos la mezcla en el mortero pero no se produjo reacción química.» Ante este posible final de la actividad 2, la docente sugirió a ambos grupos que tuvieran en cuenta todos los nuevos materiales de que disponían: «Fíjense, tal vez alguno de ellos les permite intentar alguna otra cosa con la mezcla». Sólo a partir de dicha indicación procedieron al calentamiento.

Luego de la experiencia, la pregunta 6 fue contestada correctamente por todas las alumnas, quienes reconocieron el cambio químico porque el nuevo sistema material «fue resistente a la separación del hierro con el imán».

Las preguntas 7 y 8 apuntaban a indagar cómo las alumnas fundamentaban sus respuestas, utilizando el lenguaje verbal y la esquematización mediante otros lenguajes.

Pregunta 7: Caracterice y fundamente el fenómeno producido.

El 94\% de las alumnas contestó la pregunta 7 caracterizando el fenómeno como una reacción química por la «producción de una nueva sustancia». Así, contestaron correctamente desde el lenguaje verbal, fundamentando sus respuestas en el cambio percibido desde el nivel macroscópico.

Pregunta 8: Realizar un esquema que muestre el fenómeno producido al calentar una mezcla de azufre y hierro.

Nuevamente, para este grupo de alumnas, se repitió la categorización que mostramos en el análisis a la respuesta de la pregunta 2.

La mitad del grupo A dibujó tres etapas (Fig. 7a): una primera etapa con un tubo de ensayo, sostenido por la pinza de madera, con un sombreado que representa la «nueva sustancia formada». Debajo del tubo hay un mechero encendido. La siguiente etapa muestra el mismo tubo de ensayo, con idéntico sombreado, inmerso en un vaso de precipitados con agua, y la última etapa muestra el mismo tubo de ensayos con igual sombreado, quebrado. Estos esquemas se relacionan con la secuencia experimental; nuevamente, la representación «esquemática» fue interpreta- 
da como un dibujo del protocolo del procedimiento macroscópico.

El $20 \%$ de las alumnas eligió un círculo para representar una de las sustancias y una cruz para la otra, sin señalar qué representaba cada símbolo, y dibujó en forma de ecuación una «suma» del círculo y la cruz que, mediante una flecha, se transforma en una «nueva entidad» consistente en un círculo y una cruz contiguos (Figs. 7b y 7c). La mitad de estas alumnas, expresó alguna noción de ajuste respecto al número de partículas, como puede verse en la figura $7 \mathrm{~b}$ (la representación del tipo ecuación ya se había visto en clases anteriores, al enseñar formación de iones y enlaces iónicos).

El $30 \%$ de las alumnas dibujó un tubo de ensayo con un sombreado oscuro, que representaría la nueva sustancia, sobre el cual aparecen cruces en algunos esquemas y puntos en otros (Fig. 7d), ubicándose en lo que hemos denominado nivel semiparticulado de representación (Fig. 3).

\section{Respuestas a las preguntas 5-8 para el grupo B}

La presencia del mechero fue un disparador inmediato para los alumnos del grupo B sobre la posibilidad de inducir cambios en la mezcla por la acción del calor. Los alumnos solicitaron la demostración y la docente procedió a realizar la experiencia de calentamiento en tubo de ensayo con la mezcla azufre e hierro. Las respuestas al cuestionario individual fueron las siguientes.
Pregunta 5: ¿Es posible producir una reacción química entre el azufre y el hierro? ¿Por qué?

- «Sí, porque al darles energía calórica comienzan a moverse más rápidamente hasta unirse y formar una nueva sustancia.» $(43 \%)$

- «Sí, porque se les da energía calórica, que es potencial que está almacenada en las partículas.» (5\%)

- «Sí, porque al calentar se forma una nueva sustancia.» (52\%)

El $100 \%$ de las respuestas fue afirmativo y, a diferencia del grupo A, estos alumnos hicieron referencia-correcta o incorrectamente- mediante lenguaje verbal a procesos de nivel simbólico.

Pregunta 6: ¿Cómo procedería para verificar lo que respondió en la actividad anterior?

Las respuestas fueron:

- «Viendo si las propiedades del azufre y del hierro son las mismas, comprobamos que no son las mismas, es una sustancia con propiedades totalmente diferentes.» $(13 \%)$

- «Coloco la mezcla en el tubo de ensayo y caliento.» (69 \%)

- «Le aplico algún tipo de energía.» (4 \%)

- «Hay que dar energía para producir la fusión.» (9\%)

En contraposición con las respuestas dadas por las alumnas del grupo A, que eran sencillas y correctamente basadas en la pérdida de la propiedad de ferromagnetismo del hierro, aquí las respuestas muestran dispersión en las ex-

Figura 7

Esquemas presentados por las alumnas del grupo A para el cambio químico ocurrido entre hierro y azufre (respuestas a la pregunta 8 de la actividad 2).

Figura a

Nivel Macroscópico<smiles>C1=CC2CC2(N(c2ccccc2)c2ccccc2)C=C1</smiles>
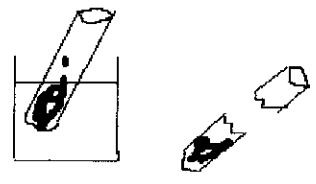

$(50 \%)$

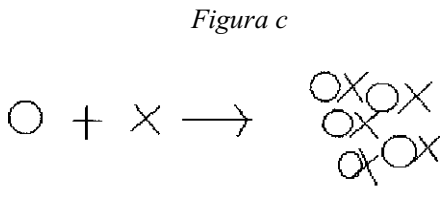

Nivel Submicroscópico (10\%)
Figura $b$

Nivel Submicroscópico

$0+x \rightarrow 0 x$

$(10 \%)$

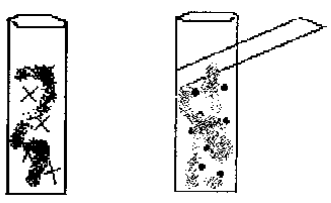

Nivel Semi-particulado

(30\%) 
plicaciones. En este caso, las expresiones vertidas mediante lenguaje verbal (Fig. 6) no resultaron lo suficientemente precisas; los códigos utilizados involucraron palabras y construcciones sintácticas de referencia conceptual ambigua. Por ejemplo, podría indagarse:

- ¿Qué significa calentar o aplicar algún tipo de energía?

- ¿A qué se refieren con fusión? ¿Acaso estos alumnos tienen la representación mental sobre la nueva sustancia como si se tratara de algún tipo de aleación, como un producto derivado de la «fusión-solidificación» de los reactivos? ¿Confunden un cambio químico con un cambio físico?

Esta situación nos lleva a reflexionar nuevamente sobre el uso de códigos para los diferentes tipos de lenguajes simbólicos. En este caso, se trata del lenguaje verbal, con el cual los expertos significamos conceptos unívocos mediante el uso de términos precisos. Diferentes sintaxis remiten a representaciones mentales alternativas. Por ejemplo, la afirmación «hay que dar energía para producir la fusión» (expresada por el $9 \%$ de los alumnos) tiene un formato sintáctico correcto en el marco del tema punto de fusión; sin embargo, no es correcta en el contexto de la reacción entre el azufre y el hierro. ¿Cómo descubrir cuál es la representación mental, la significación conceptual subyacente, que tienen esos alumnos?

Sólo podemos concluir que el lenguaje verbal utilizado por estos alumnos, entusiasmados en el área de química, exhibe una cantidad de códigos y formatos sintácticos que un experto en química no calificaría como correctos.

Esta conclusión se vio reforzada al analizar las respuestas a la pregunta 7 .

Tabla III

Respuestas de los alumnos del grupo B a la pregunta 7.

\begin{tabular}{|l|c|}
\hline Caracterización y fundamentación & Porcentajes \\
\hline $\begin{array}{l}\text { Reacción química porque hubo choques } \\
\text { efectivos con energía suficiente. }\end{array}$ & $26 \%$ \\
\hline $\begin{array}{l}\text { Reacción química. Unión química: } \\
\text { cambia la sustancia. }\end{array}$ & $29 \%$ \\
\hline $\begin{array}{l}\text { Unión iónica porque se une un metal } \\
\text { con un no-metal. }\end{array}$ & $11 \%$ \\
\hline $\begin{array}{l}\text { Reacción exotérmica porque necesita energía } \\
\text { para cambiar el sistema }\end{array}$ & $28 \%$ \\
\hline $\begin{array}{l}\text { Sistema homogéneo químico debido a que } \\
\text { al calentar el azufre y el hierro se forma } \\
\text { una sola sustancia }\end{array}$ & $2 \%$ \\
\hline \begin{tabular}{l} 
No contesta \\
\hline
\end{tabular} & $4 \%$ \\
\hline
\end{tabular}

Pregunta 7: Caracterizar y fundamentar el fenómeno producido.

En la tabla III se muestra la variada gama de sintaxis utilizada por los alumnos del grupo B para explicitar sus conclusiones mediante el lenguaje verbal.

El $26 \%$ de los alumnos respondió haciendo referencia a la teoría de las colisiones, que había sido mencionada por la docente durante la demostración. El 40\% justificó apelando al tema de las uniones químicas que habían sido enseñadas con anterioridad. Un $28 \%$ de los alumnos empleó incorrectamente la palabra exotérmica, que no fue utilizada durante el curso de química ${ }^{5}$. El $2 \%$ puede corresponderse con una confusión entre «fusión» y «reacción química», tal como se comentó más arriba.

Pregunta 8: Realizar un esquema que muestre el fenómeno producido al calentar «una mezcla de azufre y hierro».

La pregunta apuntaba a indagar qué tipos de representaciones mentales de nivel submicroscópico manejan y significan los alumnos, que se habían expresado verbalmente según lo señalado en la tabla III.

Ningún alumno interpretó el esquema desde un nivel macrosópico. El 13\% de los alumnos remitió a un nivel semiparticulado (Fig. 3), al dibujar un círculo con gran cantidad de puntos negros bien remarcados representando el aspecto macroscópico de la nueva sustancia formada (un sólido negro) y, simultáneamente, mostrando códigos típicos del nivel particulado.

El 87\% de los alumnos dibujó esquemas que se corresponden con el nivel submicroscópico de Johnstone, según se muestra en la figura 8. Es interesante, nuevamente, analizar los tipos de códigos utilizados para interpretar el fenómeno en términos de lenguaje gráfico.

El 5\% de los alumnos dibujó sólo círculos vacíos, sin color ni etiqueta. ¿Se trata de representar la nueva sustancia formada? ¿Se trata de una nueva partícula diferente de las anteriores? (Fig. 8a). El 8\% dibujó círculos y cruces sobre ellos pudiendo ser dicho esquema más representativo de una fusión, como solución homogénea, que de una transformación química (Fig. 8b). Apenas un 3\% dibujó una cruz dentro de un círculo dando idea de una unión de las partículas de ambas sustancias originales en una relación uno a uno (Fig. 8c). El $16 \%$ de los alumnos dibujó círculos superpuestos que encierran letras $\mathrm{A}$ y $\mathrm{H}$, un tipo de esquema que tampoco permitiría diferenciar el cambio químico de un producto de fusión. El $26 \%$ de los alumnos realizó un esquema similar al descripto anteriormente, pero empleando los símbolos químicos para el azufre y el hierro (Fig. 8e). El 29 \% restante escribió los símbolos del hierro y del azufre unidos y encerrados en un óvalo, representando con ello la unión en la misma partícula, probablemente representando la nueva sustancia formada mediante la unión del azufre y el hierro (Fig. 8f).

Esta rica variedad de códigos para representar lo ocurrido a nivel submicroscópico nos está indicando la necesidad de pactar o consensuar con los alumnos qué se pretende 
Figura 8

Esquemas presentados por el $87 \%$ de los alumnos del grupo B para representar el cambio químico ocurrido entre hierro y azufre.

Figura 8a

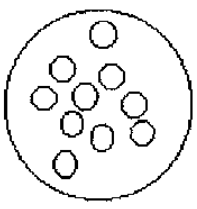

$(5 \%)$
Figura 8b

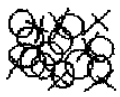

(8\%)

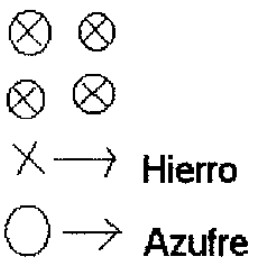

(3\%)
Figura 8d

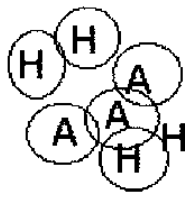

$(16 \%)$
Figura 8e

(26\%)

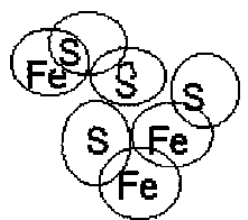

Figura $8 f$

$(29 \%)$

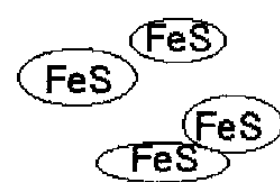

representar, y con qué símbolos. Ante la variedad de códigos utilizados por los alumnos (Fig. 8) cabe reflexionar, ya que somos expertos, qué tipo de representación esquemática a nivel particulado daríamos como «respuesta correcta» a la pregunta formulada, considerando las alternativas presentadas en la figura 9. Este aspecto, generalmente relegado, que involucra la toma de conciencia sobre las convenciones sintácticas estipuladas para las representaciones mediante esquemas de partículas, es crítico para que se establezca una adecuada comunicación entre el docente y los alumnos. Los expertos sabemos que la elección de códigos para el lenguaje gráfico mediante esquemas de partículas conlleva siempre simplificaciones, pero es muy probable que el alumno perciba las representaciones gráficas como «verdaderas y únicas», aunque no le signifiquen demasiado.

\section{CONCLUSIONES}

La comunicación entre el profesorado y el alumnado de ciencias naturales encuentra una serie de dificultades.

Una de las dificultades está asociada a la brecha que se produce entre el lenguaje cotidiano y el lenguaje cientifico erudito (Galagovsky, Bonán y Adúriz-Bravo, 1998;
Figura 9

Diferentes posibilidades de representar, mediante esquemas de partículas, la estructura del compuesto sulfuro ferroso y de su proceso de obtención a partir de azufre y hierro.
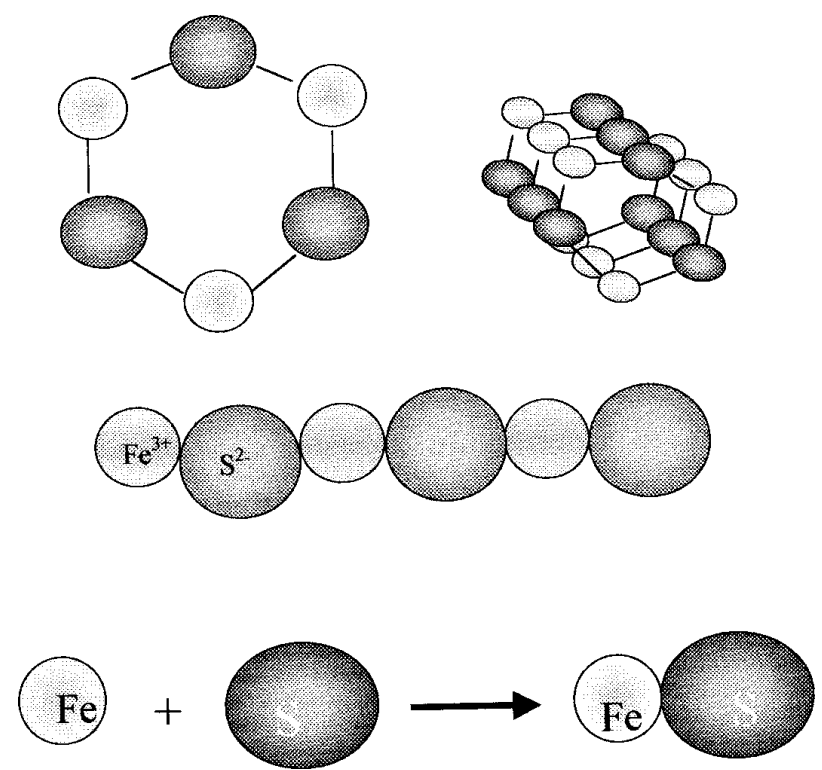

ENSEÑANZA DE LAS CIENCIAS, 2003, 21 (1) 
Galagovsky y Adúriz-Bravo, 2001), ya que la apropiación del lenguaje científico es un proceso gradual y contextualizado (Lemke, 1997; Izquierdo y Sanmartí, 1998, 1999).

El discurso erudito es, en realidad, una información explícita que utiliza múltiples lenguajes. Cada lenguaje tiene sus propios códigos y formatos sintácticos aceptados y compartidos por los expertos, pero estas explicitaciones generalmente se omiten durante las explicaciones dadas en clase.

El aspecto sintáctico es el aspecto explícito de un mensaje o de una información; es aquella estructura de la comunicación que todos pueden apreciar o percibir. El aspecto semántico del lenguaje es, en cambio, la significación a la que cada sujeto remite cada parte o el todo de una comunicación. La significación que se da a las palabras, a las oraciones o a los dibujos es algo que ocurre «dentro de la cabeza» de los sujetos. Esta destreza cognitiva no se puede «ver» ni percibir mediante los sentidos.

Cuando un profesor escribe una ecuación, o un gráfico, o una fórmula, estos símbolos tienen sentido para él y para otros expertos En cambio, para un novato, esa sintaxis pueden no tener ningún tipo de significación o bien puede darle otra significación, desde su «buen saber y entender», desde su sentido común, desde su conocimiento cotidiano.

Frecuentemente los alumnos aprenden de memoria las sintaxis que «dictan los profesores», con sus códigos implícitos y, luego, los expresan frente a la demanda evaluativa del profesor. Sin embargo, ¿qué significación real le otorga cada alumno a dichas construcciones sintácticas?

La otra dificultad la centramos en las grandes diferencias entre las diversas representaciones mentales idiosincráticas que construyen los alumnos acerca del mundo natural y las correspondientes representaciones mentales que tienen los expertos sobre los mismos temas. La movilidad mental entre los niveles de representación macroscópico y simbólico y, dentro del nivel simbólico, la equivalencia entre las explicaciones dadas en diferentes tipos de lenguajes (Fig. 6) configuran lo que denominaríamos las destrezas cognitivas del experto (Fig.10).

El alumno, para llegar a compartir las destrezas cognitivas del experto -aunque sea en un pequeño tema-debe adquirir dicha movilidad representacional, pero a partir del lenguaje cotidiano, con palabras que tienen su significación desde lo cotidiano. Palabras nuevas no encuentran fácilmente un anclaje de significación. Los alumnos pueden estudiar de memoria oraciones enteras -construcciones sintácticas- o bien pueden imaginarse, es decir, representarse mentalmente cosas diferentes a las que el docente esperaba.

La figura 10 muestra esquemáticamente las diferencias entre las destrezas cognitivas de expertos y novatos. Allí se evidencia que sólo los niveles sintácticos del lenguaje son explícitos y son el vínculo real de comunicación entre los docentes y los alumnos.

En este trabajo hemos mostrado que, por un lado, el grupo de las alumnas tendía a responder utilizando sintaxis simples y correctas desde el lenguaje verbal, pero mostraba dificultades en otro tipo de representaciones basadas en lenguaje gráfico. Por otro lado, el grupo de alumnos mostraba mayor despliegue representacional tanto en lenguaje gráfico como verbal, siendo mucho menos precisos que las alumnas en este último caso. Asirse a respuestas «premoldeadas» repitiendo sintaxis dadas como correctas por el profesor o animarse a expresar lo que se entendió, a riesgo de equivocarse en la precisión de lo expresado o en lo comprendido, implica reflexionar sobre el papel que juegan los errores durante el aprendizaje. Y esta reflexión cabe tanto a alumnos como a docentes pero, sin duda, el

Figura 10

Comparación de representaciones mentales y lenguaje entre expertos y novatos. Sólo los aspectos sintácticos del lenguaje son explícitos y, por lo tanto, información comunicable entre expertos y novatos.

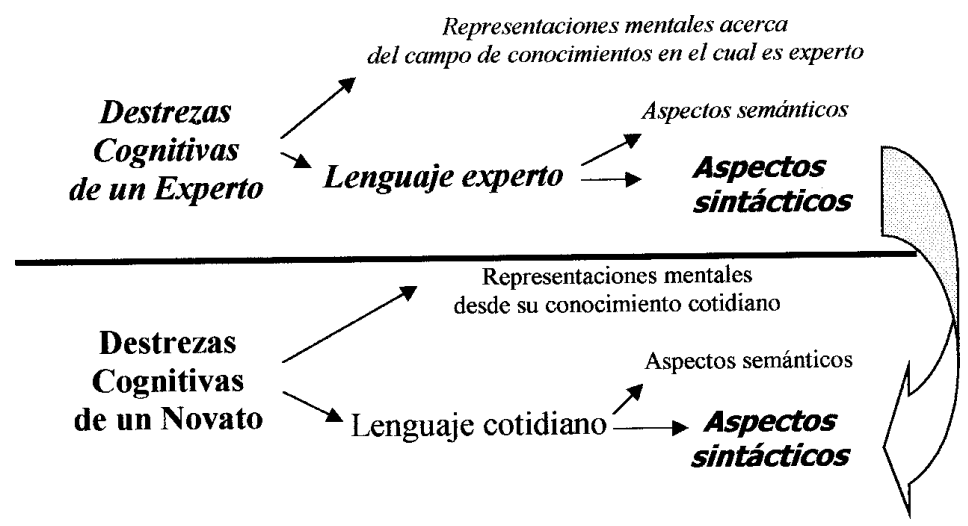


último control en esta opción lo tiene el docente, quien marcará tendencias definitivas con su sistema de evaluación y calificación (Galagovsky, 1993).

Las representaciones mentales acerca de un tema serían grados complejos de significación, combinados, expresados mediante lenguajes alternativos, complementarios y convergentes sobre los mismos conceptos científicos. La mente del experto tiene movilidad entre diferentes niveles representacionales. Aprender, para el novato, consistirá en llegar a compartir las significaciones y representaciones mentales de los expertos.

Enseñar no es sólo «decir», ya que el conocimiento no se «transmite» de la cabeza del experto a la del novato. Lo que tiene en la cabeza el experto debe hacerse explícito mediante determinadas expresiones, utilizando diferentes lenguajes, consensuando códigos, favoreciendo la articulación entre esos formatos sintácticos.

La actividad de consensuar las representaciones, el qué y el cómo de las mismas, ocurre habitualmente en el seno de la comunidad científica. Por lo tanto, destacaríamos la necesidad de que la práctica pedagógica dispusiera del tiempo necesario para consensuar representaciones, como una actividad modelo propia de la ciencia erudita. Los alumnos, trabajando en forma similar a los expertos, podrían proponer códigos, especialmente para la representación en el nivel simbólico particulado y luego reflexionar sobre la pertinencia de los códigos utilizados. Asimismo, la utilización de diversos lenguajes, para evidenciar representaciones mentales alternativas del mismo concepto o fenómeno, podría evitar la compartimentalización y ayudar a los alumnos a integrar y otorgar movilidad a sus aprendizajes. Finalmente, llegamos a las siguientes conclusiones:

- Es necesario modificar la propuesta teórica de Johnstone acerca de los niveles representacionales de la química (Fig. 2), ya que el nivel de representación submicroscópico es, en sí mismo, un nivel simbólico mediado por un lenguaje gráfico que utiliza esquemas de partículas y que se expresa mediante códigos específicos (Figs. 4 y 6 ).

- La acción mediadora del docente es fundamental para lograr la explicitación de las representaciones mentales de los alumnos, generalmente asociadas al aprendizaje perceptual (Fig. 1) y de nivel macroscópico (Fig. 2), de tal forma que evidencie los obstáculos epistemológicos que dificultan el acceso a niveles representacionales simbólicos.

- La enseñanza debe favorecer la integración de los aspectos semánticos y sintácticos de los distintos lenguajes con que los expertos interpretan conceptualmente los fenómenos químicos.

\section{NOTAS}

${ }^{1}$ El grupo A se caracterizaba por tener baja motivación frente al aprendizaje de la asignatura química. La tendencia general que se percibía era la preferencia de sus integrantes a desarrollar un aprendizaje de tipo memorístico con el único propósito de aprobar la asignatura.

El grupo B, en cambio, se caracterizaba por tener alta motivación frente al aprendizaje de la química. Los alumnos solían participar activamente en las clases e interesarse por las demostraciones.

${ }^{2}$ En Driver (1992) se afirma que la mayoría de los estudiantes de secundaria a quienes se les presenta el modelo particulado de la materia emplean en sus descripciones y dibujos el concepto de que la materia está compuesta por partículas, en vez de considerarla continua. Sin embargo, cuando utilizan conceptos relativos a las partículas, tienden a pensar que las partículas no son invariables en sí mismas y a menudo consideran que éstas poseen las mismas propiedades macroscópicas que la sustancia de la que forman parte. Por tanto, las ideas relativas a las partículas, por sí mismas, no concuerdan necesariamente con las referidas a la conservación de la materia, ya que las partículas podrían fundirse, encogerse, dilatarse, colorearse, etc.

${ }^{3}$ La diferencia entre cambios físicos y químicos había sido tratada en el primer mes de clases mediante las definiciones relativas a la variación o permanencia de las propiedades de las sustancias involucradas en los fenómenos analizados. El hincapié estuvo puesto en las características de reversibilidad o irreversibilidad de ambos tipos de cambios.

${ }^{4}$ La idea fue generar un conflicto cognitivo con la pregunta 5 , ya que ésta cuestionaba la certeza de los alumnos de estar frente a la presencia de una mezcla estable y separable (comprobado reiteradamente en las condiciones ambientales en que ellos estaban trabajando).

${ }^{5}$ Probablemente el término surgió por asociación con procesos metabólicos estudiados en la asignatura de biología, donde se habían trabajado transformaciones que requieren energía o la liberan. 


\section{REFERENCIAS BIBLIOGRÁFICAS}

BEN ZVI R., EYLON, B. y SILBERSTEIN, J. (1982). Student conception of gas and solid difficulties to function in a multiatomic context, part. II. Comunicación presentada al National Association for Research in Science Teaching Conference, Lake Geneva, WI.

BEN ZVI. R y COHEN, I. (1992). Improving student achievement in the topic of chemical energy by implementing new learning materials and strategies. Int. J. Sci. Edu., 1992, 14(2), pp. 147-156.

BOUJAUDE, S.B. (1991). A suty the nature as student's understandings about the concept of burning. Journal of Research in Science Teaching, 28(8), pp. 869-704.

COLÁS BRAVO, M. y BUENDÍA EISMAN, L. (1994). Investigación educativa. Sevilla: Alfar.

DRIVER, R. (1981). Pupils' alternative frameworks in science. European J. Sci. Educ., 3(1), pp. 93-101.

DRIVER, R., GUESNE, E. y TIBERGHIEN, A. (1992). Ideas cientificas en la infancia y la adolescencia. Madrid: Ediciones Morata.

FRIEDEL, A.W. y MALONEY, D.P. (1992). An exploratory, classroom-based investigation of students'difficulties with subscripts in chemical formulas. Science Education, 76, pp. 65-78.

GABRIELA, T.C., RIBEIRO, M., COSTA PEREIRA, D. Y MASKILL, R. (1990). Reaction and spontaneity: The influence of meaning from everyday language on fourh year undergraduates interpretations of some simple chemical phenomena. Int. J. Sc. Educ., 13(3), pp. 313-319.

GALAGOVSKY, L. (1993). Hacia un nuevo rol docente. Una propuesta diferente para el trabajo en el aula. Buenos Aires: Troquel.

GALAGOVSKY, L. (1999). Redes conceptuales: aprendizaje, comunicación y memoria. (2a. ed. Buenos Aires: Lugar Editorial.

GALAGOVSKY, L. y ADÚRIZ-BRAVO, A. (2001). Modelos y analogías en la enseñanza de las ciencias naturales. El concepto de modelo didáctico analógico. Enseñanza de las Ciencias, 19(2), pp. 231-242.

GALAGOVSKY, L., BONÁN, L. Y ADÚRIZ-BRAVO, A. (1998). Problemas con el lenguaje científico en la escuela. Un análisis desde la observación de clases de ciencias naturales. Enseñanza de las Ciencias, 16(2), pp. 315-321.

GALAGOVSKY, L., MUÑOZ, J.C. (2002). La distancia entre aprender palabras y aprehender conceptos. El entramado de palabras-concepto (epc) como un nuevo instrumento para la investigación. Enseñanza de las Ciencias, 20(1), pp. 29-45.

IZQUIERDO, M. (1999). (ed.) Aportación de un modelo cognitivo de ciencia a la enseñanza de las ciencias. Enseñanza de las Ciencias, núm. extra.
IZQUIERDO, M. y SANMARTÍ, N. (1998). Ensenyar a llegir i a escriure textos de ciències de la naturalesa, en Jorba, J., Gómez y Prat, À. (comps.). Parlar i escriure per aprendre. Us de la llengua en situació d'ensenyament-aprenentatge de les árees curriculars. Bellaterra: ICE-UAB.

JOHNSTONE, A.H. (1982). Macro and micro chemistry. School Science Review, 64 (227), pp. 377-379.

JOHNSTONE, A.H. (1991). Why is science difficult to learn? Things are seldom what they seem. J. Computer Assisted Learning, 7, pp 75-83.

JOHNSTONE, A.H. (1997). Chemistry teaching. Science or Alchemy? J. Chem. Ed., 74(3), pp. 262- 268.

JOHNSTONE, A.H.(1999). The nature of chemistry. Education in Chemistry, pp. 45-47.

LEMKE, J. (1997). Aprender a hablar ciencia. Barcelona: Paidós. (Edición original de 1993)

MALONEY, D.P. y FRIEDEL, A.W. (1991). Students'difficulties with subscripts in chemical formulas. Comunicación presentada a National Association for Research in Science Teaching Conference. Fontana, WI.

MAYER, R.E. (1985). El futuro de la psicología cognitiva. Buenos Aires: Alianza Editorial.

NIAZ, M. y LAWSON, A.E. (1985). Balancing chemical equations: The rol of developmental level, mental capacity and cognitive style. Comunicación presentada a National Association for research in Science Teaching Conference. Lake Geneva, WI.

RODRÍGUEZ, M.A. (2001). «Un estudio sobre la vinculación entre el lenguaje utilizado en clase y las representaciones construidas por los estudiantes durante el aprendizaje del tema reacciones químicas.» Tesis de licienciatura en enseñanza de la química. Universidad Nacional de General San Martín. Buenos Aires. Argentina.

SOLSONA, N. y IZQUIERDO, M. (1998). La construcción del concepto de cambio químico. Los modelos teóricos, un instrumento para su análisis, en Banet y de Pro (eds.). Investigación e Innovación en la Enseñanza de las Ciencias, pp. 327-335. Murcia.

SOLSONA, N., IZQUIERDO, M. y DE JONG, O. (2001). Un estudio de la evolución de los perfiles conceptuales del alumnado sobre la reacción química. Revista de Educación en Ciencias, Boletín de Novedades CREDI-OEI, 51, pp 4447. Sta. Fe de Bogotá. Colombia.

TABER, K.S (1998). The sharing-out of nuclear attraction: or 'I can't think about physics in chemistry'. Int.J.Sci.Edu., 20(8), pp. 1001-1014.

YARROCH, W.L. (1985). Student understanding of chemical equation balancing. Journal of Research in Science Teaching, 22(5), pp. 449-459. 